\section{JTI}

JOURNAL OF

TRAUMA AND INJURY

\title{
Analysis of the Importance of Sacroiliac Joint Fractures as a Prognostic Factor of the Patients with Pelvic Fractures
}

\author{
Yeon-Uk Ju, M.D. ${ }^{1}$, Jun-Min Cho, M.D. ${ }^{1}$, Nam-Ryeol Kim, M.D. ${ }^{1}$, \\ Kyung-Bum Lee, M.D., Ph.D. ${ }^{1}$, Jin-Kak Kim, M.D. ${ }^{2}$, Jong-Keon Oh, M.D., Ph.D. ${ }^{2}$ \\ Departments of ${ }^{1}$ General Surgery, ${ }^{2}$ Orthopedic Surgery, Korea University Guro Hospital, Seoul, \\ Korea
}

Purpose: The diagnosis of pelvic fractures pattern has become to be essential in the decision making of treatment modality and reducing morbidity and mortality in multiple trauma patients. Sacroiliac joint (SIJ) disruption can cause life-threatening massive arterial bleeding. This study aimed to determine a method of predicting the prognosis and treatment direction with pelvis X-ray alone in the emergency room. We investigated whether SIJ disruption can be used alone as a poor prognostic factor.

Methods: We analyzed the medical records and radiologic examination results of 167 patients with pelvic fractures from January 1, 2015 to December 31, 2016 retrospectively. Patients with pathologic fractures, thoraco-abdominal bleeding, and acetabulum fractures and pediatric patients $(n=63)$ were excluded. Factors related to the clinical manifestations and treatments, such as transfusion and surgery, were statistically compared. Results: The cross-sectional analysis showed that there was no correlation between SIJ injury and sex; there were statistically significant relationships between occurrences of shock, conjoined fractures, transfusion, and surgeries. The hospitalization period and partial thromboplastin time and prothrombin time values increased. The logistic regression analysis showed that when an SIJ injury occurred, blood transfusion and hypotension possibilities increased.

Conclusions: When pelvic fractures occur near the SIJ, blood transfusion and shock possibilities increase. Physicians must be aware of the high severity and poor prognosis of such fractures when these are diagnosed in the emergency room. And furthermore, the physician has to predict and prepare the intensive care and multidisciplinary approaches.

Keywords: Iliac artery; Multiple trauma; Shock; Pelvic bones 


\section{INTRODUCTION}

Traumatic pelvic fractures are associated with high mortality and morbidity rates owing to massive hemorrhage despite the progress of medical treatments, and there are many debates on the application of treatment modalities [1-5]. The current treatment of patients with pelvic ring fractures varies to obtain good outcomes. Pelvic fixation combined with radiologic angiography and embolization, preperitoneal pelvic packing (PPP) and resuscitative endovascular balloon occlusion of the aorta are the multidisciplinary approaches using variable treatment modalities $[5,6]$. Nonetheless, hemodynamically unstable pelvic fractures must be rapidly diagnosed and classified in the emergency department; predicting massive arterial bleeding and determining the initial treatment strategy play a crucial role in decreasing the mortality rates $[7,8]$.

There are various classifications of pelvic fracture according to injury mechanism, clinical features, and shape of the fracture itself. Most popular Young-burgess classification is refined according to the stability of pelvic fractures. However, most of these classifications are complex and have limitations in predicting the prognosis of patients [9]. Injury in the branches of the internal iliac artery (IIA) is the most common cause of massive hemorrhage $[2,4,8]$. The IIA, which accounts for most of the blood supply to the pelvis, passes through the lower part of the sacroiliac joint (SIJ). As a result, the presence of SIJ disruption could be associated with injuries of IIA and influence the severity of injury. Based on this concept, the purpose of this study is to find simple prognostic factor of the pelvic ring injuries. We investigated whether SIJ disruption is an independent variable affecting the treatment and prognosis of the pelvic ring injury.

\section{METHODS}

From January 1, 2015 to December 31, 2016, 167 patients with pelvic fractures were referred to Korea University Guro Hospital. Of these, we excluded 19 patients with torso hemorrhage necessitating neurosurgical and thoracoabdominal surgeries, eight patients with pathologic fractures, nine patients aged under 15 years, four patients from other hospitals after 24-hour treatments, three death-on-arrival (DOA) patients, and 20 patients with acetabular fractures without pelvic ring injury. These patients were excluded because the purpose of this study was to minimize the effect of other conjoined multiple injuries and to identify the clinical features of pelvic fracture itself. Finally, 104 patients remained and were divided into the SIJ injury group as defined in group A and non-SIJ injury group as group B (Fig. 1).

The patients received blood transfusion when their vital signs became unstable even after rapid infusion of $2 \mathrm{~L}$ of normal saline and when their hemoglobin levels dropped to below $7 \mathrm{~g} / \mathrm{dL}$. Shock is defined by a systolic pressure of less than $90 \mathrm{mmHg}$. Early pelvic binder was applied to the center of the greater trochanter of both femurs, and external fixation, PPP, and angioembolization were performed selectively.

The study parameters were age, sex, prothrombin time (PT) and partial thromboplastin time (PTT) values, SIJ injury, systolic blood pressure, shock, combined fractures, blood transfusion, length of hospital stay, and hemoglobin level. The SIJ lesions were evaluated by reviewing pelvic X-ray and pelvic computed tomography (CT) images at admission. The disruption of the anterior ligament and posterior ligament were all included. The lowest measurement recorded for the blood pressure and hemoglobin level after admission was used.

Statistical analysis was performed using the independent $t$-test and chi-square test and logistic regression analysis using the SPSS version 20 (IBM Corp., Armonk,

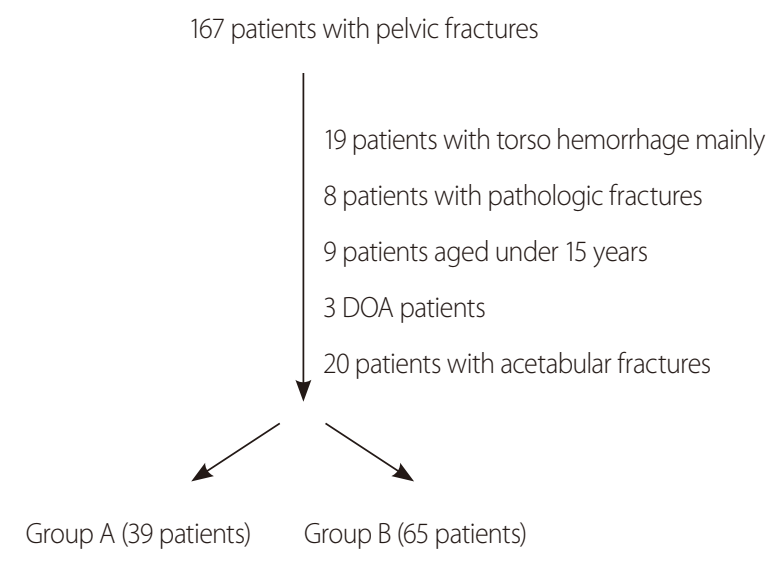

Fig. 1. Patient selection method flow gram. DOA: dead on arrival. 
NY, USA). A p-value of less than 0.05 was defined as statistically significant.

\section{RESULTS}

The distribution of the patients with pelvic ring injury was same according to the gender (men and women: both $\mathrm{n}=52)$. There were 22 men $(56 \%)$ in group A and 30 men $(46 \%)$ in group B. Their average age was 56.4 years. The injuries were more commonly observed in the middle-aged patients. The most common cause of injuries was traffic accidents. Pedestrian traffic accidents were more common in group A. Injury pattern classification by Young-Burgess and presence of accompanying fractures showed a significant difference between the two groups (Table 1).

Blood pressure was significantly higher in group B, and shock of less than 90-mmHg systolic pressure occurred in group A $(n=15)$. Among the patients with shock, angio-

\section{Table 1. Patient characteristics}

\begin{tabular}{|lccc|}
\hline & $\begin{array}{c}\text { Group A } \\
(\mathbf{n}=39)\end{array}$ & $\begin{array}{c}\text { Group B } \\
(\mathbf{n}=65)\end{array}$ & p-value \\
\hline Age (years) & $52.9 \pm 17.7$ & $28.4 \pm 22.0$ & 0.070 \\
Male & $22(56)$ & $30(46)$ & 0.320 \\
Mechanism of injury & 39 & 65 & 0.023 \\
In-car & 11 & 20 & \\
Pedestrian & 21 & 21 & \\
Fall down & 7 & 13 & \\
Slip down & 0 & 11 & \\
Young-Burgess classification & & & $<0.001$ \\
LC1 & 0 & 61 & \\
LC2 & 13 & 4 & \\
LC3 & 6 & 0 & \\
AP1 & 4 & 0 & \\
AP2 & 10 & 0 & \\
AP3 & 3 & 0 & \\
VS & 3 & 0 & \\
Extremity fractures & 28 & 26 & 0.001 \\
\hline
\end{tabular}

Values are presented as mean \pm standard deviation or number (\%). LC: lateral compression, AP: anteroposterior compression, VS: vertical shear. graphic embolization was performed in five patients and PPP in one patient. Transfusion was performed in $10 \mathrm{pa}-$ tients (67\%) and massive transfusion requiring more than 10 units in one patient. In group A, the occurrence of shock was significantly higher, indicating that SIJ damage and shock were closely related.

The mean hemoglobin level was $11.3 \mathrm{~g} / \mathrm{dL}$ in group A and $11.9 \mathrm{~g} / \mathrm{dL}$ in group $\mathrm{B}(p=0.629)$. However, 24 patients (61\%) had hemoglobin levels below $10 \mathrm{~g} / \mathrm{dL}$ in group A, and 15 patients received blood transfusion. The coagulation factor test results for PT and PTT significantly increased in group A. The area under the curve of the receiver operating characteristic curve was 0.727 and 0.728 for PT and PTT, respectively. The PT and PTT values in group A were correlated with the sensitivity and specificity of the SIJ lesion compared to the hemoglobin level (Fig. 2). These laboratory data can be used as a prognostic factor.

The mean hospitalization period was 25.69 days in group A and was admitted for up to 60 days. The patients in group B were hospitalized in an average of 6.91 days. The patients with minor injuries were discharged after the evaluation. These stable 14 patients had no blood test results and excluded in statistical analysis.

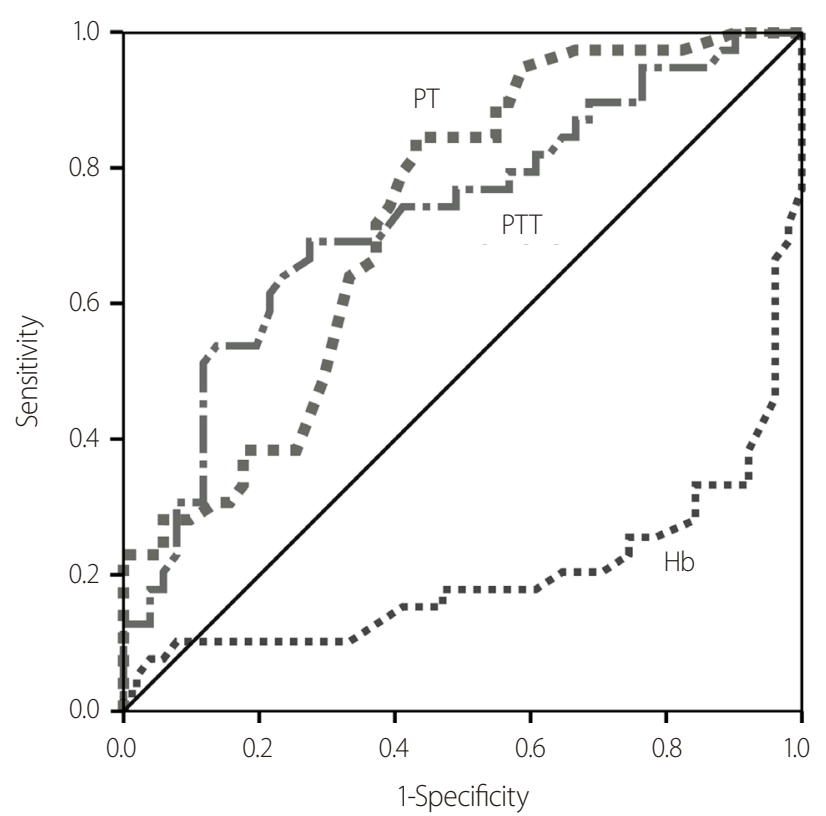

Fig. 2. ROC curve of SIJ disruption and variables. ROC: receiver operating characteristic, Hb: hemoglobin, PTT: partial thromboplastin time, PT: prothrombin time, SIJ: sacroiliac joint. 
Thirty-eight patients (58\%) required outpatient treatment in group B. The mean injury severity score was 20.93 in group A, and the incidence of severe trauma exceeding 15 points was significantly higher in group A than in group B. Pelvic fixation was performed in 31 patients in group A and 10 patients in group $B$, which was statistically significant $(p<0.01)$. There was also a significant increase in the number of limb fractures with pelvic fractures in group A. Angiography and embolization were performed in five patients in group A, and there were shock state in that patients. PPP was performed in four patients (Table 2).

During the study period, there were five pelvic fracture-associated deaths, two hemoperitoneum cases, two cerebral hemorrhage cases, and two DOA cases. However, there was no mortality in the enrolled patients with pelvic fracture only.

We performed a logistic regression analysis of the SIJ damage and analyzed the possibility of shock and blood

Table 2. Comparisons of the laboratory and clinical data

\begin{tabular}{|lccc|}
\hline & $\begin{array}{c}\text { Group A } \\
(\mathbf{n}=\mathbf{3 9})\end{array}$ & $\begin{array}{c}\text { Group B } \\
(\mathbf{n}=65)\end{array}$ & $\boldsymbol{p}$-value \\
\hline Laboratory results & & & \\
$\quad$ Hemoglobin (g/dL) & $11.3 \pm 11.4$ & $11.9 \pm 1.4$ & 0.629 \\
PT (seconds) & $15.7 \pm 3.5$ & $14 \pm 1.1$ & 0.003 \\
PTT (seconds) & $39.4 \pm 6.6$ & $34.9 \pm 4.8$ & 0.001 \\
SBP (mmHg) & $100.38 \pm 19.4$ & $127.54 \pm 15.5$ & $<0.001$ \\
ISS & $20.93 \pm 9.7$ & $8.73 \pm 4.7$ & 0.002 \\
Transfusions & 15 & 3 & $<0.001$ \\
LOS & $25.69 \pm 13.6$ & $6.91 \pm 9.6$ & $<0.001$ \\
Angioembolization & 5 & 0 & \\
PPP & 4 & 0 & \\
Operations (orthopedic) & 31 & 10 & $<0.001$ \\
\hline
\end{tabular}

Values are presented as mean \pm standard deviation or number. PT: prothrombin time, PTT: partial thromboplastin time, SBP: systolic blood pressure, ISS: injury severity score, LOS: length of stay, PPP: preperitoneal packing.

Table 3. Possibility after SIJ injury

\begin{tabular}{|lccrc|}
\hline Variable & B & SE & OR & $\boldsymbol{p}$-value \\
\hline Transfusion & 3.009 & 1.205 & 20.190 & 0.013 \\
SBP & 0.119 & 0.029 & 1.127 & $<0.001$ \\
\hline
\end{tabular}

SIJ: sacroiliac joint, SE: standard error, OR: odds ratio, SBP: systolic blood pressure. transfusion. It was found that the possibility of transfusion and hypotension increased when a SIJ damage occurred (Table 3).

\section{DISCUSSION}

Pelvic fractures are rare fractures with an incidence rate of $3 \%$ to $8 \%$. However, it is one of the important causes of non-compressible torso hemorrhage. Traumatic pelvic fractures may result in severe hemorrhage and death. The pelvic fracture-related mortality rate has been reported to be up to $15 \%$ [10-16]. The treatment modality depends on the injury mechanisms, clinical features, and radiological fracture patterns [11]. Venous bleeding is common and occurs mainly in the presacral plexus. Most of these cases are hemodynamically stable due to retroperitoneal tamponade, which can stop low-pressure venous bleeding from the bones and veins. Although arterial bleeding is rare, high-pressure arterial bleeding can exceed the retroperitoneal pressure and result in massive hemorrhage. Such an event is diagnosed as blushing on CT or angiography. Scemama et al. [12] reported that $75 \%$ of the cases of hemorrhage requiring angiography in the presence of contrast leakage were detected on CT scan. Angiography and embolization are effective in stopping arterial bleeding $[8-11,17,18]$. In the early 1970s, direct hematoma removal and exploration for bleeding control were criticized by numerous studies, which report that pelvic exploration could not control bleeding and that the mortality rate could increase. Most venous hemorrhages could be managed non-operatively. When arterial bleeding was combined with pelvic fracture, hemodynamic instability was frequent, and hemostasis was required [12]. Young et al. [2] reported that a particular pelvic fracture pattern is associated with a major ligamentous disruption related to arterial bleeding. The correlation between the particular type of pelvic fracture and occurrence of hypotension can be predicted as arterial bleeding. In the present study, Group A, which is more likely to have arterial bleeding, had overlapping classifications with lateral compression (LC) 2, LC3, anteroposterior compression (AP) 2, AP3, and vertical shear (VS) types according to the Young-Burgess Classification and group B with LC1 and AP1. There 
were significant bleeding, blood transfusion, shock, and severe impairment in group A.

The presence of SIJ disruption can be diagnosed in simple X-ray images. Physician can judged and predicted the occurrence of massive hemorrhage; further, SIJ lesions are considered a factor more commonly involved in the severity of the patient condition than the absence of SIJ damage. Thus, these could be used as an indicator to predict the prognosis of patients and treat them.

Tile et al. [4] classified pelvic fractures, while adding the concept of pelvic stability, and introduced the concept of force vector. These concepts aided in the performance of external fixations for pelvic fractures. The classification system of such fractures can be used to predict hemodynamic stability, pelvic instability, and soft tissue injuries [3]. Sharpe et al. [5] reported that initial fixation not only reduced early bleeding but also had a positive impact on rehabilitation in their long-term follow-up. However, they also pointed out that inaccurate fracture patterns may lead to inappropriate fixation, which may lead to additional damage. The definitive treatment of fractures is determined on the basis of the clinical features and pattern of the fracture [3,4]. However, Sarin et al. [9] reported that the Young-Burgess classification cannot predict severe arterial bleeding and should consider age, sex, physical examination results, and injury mechanisms. In addition, there is a limit in determining the treatment and prognosis using the classification system of fractures [9]. In addition, it is not easy to determine the fracture pattern in the emergency room in a short time. The classification system of fractures needs to be continuously improved, in which clinical studies are needed. In this study, there was no significant difference in age and sex between the two groups. The higher the severity of pelvic fracture, the higher the frequency of transfusion and hypotension, and the longer the length of hospitalization owing to the increased number of complications. The presence of transfusion and hypotension suggests the poor prognosis in this study.

This study excluded other causes of non compressible torso hemorrhages and cases of thoracoabdominal and brain surgeries and included purely hemorrhagic and hemodynamic instabilities due to pelvic fractures; there were no deaths recorded in the study subjects [10]. Vaidya et al.
[7] reported a $0.2 \%$ mortality rate in isolated pelvic fractures, suggesting a poorer survival rate in the presence of multiple torso trauma. Further, they suggested that pelvic fractures accompanied with head injuries and thoracoabdominal vascular injuries require multidisciplinary approaches because of the life-threatening conditions. The reported overall mortality rates for these patients vary; however, there is a broad agreement that death as a direct result of pelvic fractures occurs in less than $1 \%$ of patients admitted due to this injury. However, the mortality rate is high in patients with pelvic fractures combined with head and thoracoabdominal injuries [12,17-21].

There are some limitations due to retrospective design of this study. Further studies of combined injury and pelvic fracture and case studies of isolated pelvic fractures are needed.

\section{CONCLUSION}

Pelvic fractures without associated injuries other than limb fractures have a relatively low mortality rate; however, the risk of mortality is high when pelvic fractures are accompanied with NCTH. When patients with SIJ injuries are diagnosed via plain X-ray, we can expect a longer hospitalization period, the possibility of shock, and the need for blood transfusion. At the same time, we should admit such patients immediately and start intensive care, including blood transfusion, pelvic fixation, and angiographic embolization. And the patients with unstable pelvic fractures should be treated via a multidisciplinary approach, instead of active orthopedic surgeries alone, because of the life-threatening torso hemorrhage mechanisms.

\section{REFERENCES}

1. Sathy AK, Starr AJ, Smith WR, Elliott A, Agudelo J, Reinert $\mathrm{CM}$, et al. The effect of pelvic fracture on mortality after trauma: an analysis of 63,000 trauma patients. J Bone Joint Surg Am 2009;91:2803-10.

2. Young JW, Burgess AR, Brumback RJ, Poka A. Pelvic fractures: value of plain radiography in early assessment and 
management. Radiology 1986;160:445-51.

3. Haq RU, Dhammi IK, Srivastava A. Classification of pelvic fractures and its clinical relevance. J Orthop Traumatol Rehabil 2014;7:8-13.

4. Tile M, Helfet DL, Kellan JF. Fractures of the pelvis and acetabulum. 3rd ed. Baltimore: Lippincott Williams and Wilkins; 2003.

5. Sharpe JP, Magnotti LJ, Gobbell WC, Huang X, Perez EA, Fabian TC, et al. Impact of early operative pelvic fixation on long-term self-reported outcome following severe pelvic fracture. J Trauma Acute Care Surg 2017;82:444-50.

6. Matsumoto J, Lohman BD, Morimoto K, Ichinose Y, Hattori T, Taira Y. Damage control interventional radiology (DCIR) in prompt and rapid endovascular strategies in trauma occasions (PRESTO): a new paradigm. Diagn Interv Imaging 2015;96:687-91.

7. Vaidya R, Scott AN, Tonnos F, Hudson I, Martin AJ, Sethi A. Patients with pelvic fractures from blunt trauma. What is the cause of mortality and when? Am J Surg 2016;211:495500 .

8. Flint L, Cryer HG. Pelvic fracture: the last 50 years. J Trauma 2010;69:483-8.

9. Sarin EL, Moore JB, Moore EE, Shannon MR, Ray CE, Morgan SJ, et al. Pelvic fracture pattern does not always predict the need for urgent embolization. J Trauma 2005;58:973-7.

10. Morrison JJ, Rasmussen TE. Noncompressible torso hemorrhage: a review with contemporary definitions and management strategies. Surg Clin North Am 2012;92:843-58, vii.

11. Cullinane DC, Schiller HJ, Zielinski MD, Bilaniuk JW, Collier BR, Como J, et al. Eastern Association for the Surgery of Trauma practice management guidelines for hemorrhage in pelvic fracture--update and systematic review. J Trauma 2011;71:1850-68.

12. Scemama U, Dabadie A, Varoquaux A, Soussan J, Gaudon C, Louis G, et al. Pelvic trauma and vascular emergencies. Diagn Interv Imaging 2015;96:717-29.

13. Yu BC, Chung M. Management strategy of bleeding pelvic fracture. Trauma Image Proced 2016;1:1-6.
14. Hauschild O, Strohm PC, Culemann U, Pohlemann T, Suedkamp NP, Koestler W, et al. Mortality in patients with pelvic fractures: results from the German pelvic injury register. J Trauma 2008;64:449-55.

15. Balogh Z, King KL, Mackay P, McDougall D, Mackenzie S, Evans JA, et al. The epidemiology of pelvic ring fractures: a population-based study. J Trauma 2007;63:1066-73; discussion 1072-3.

16. Song KH, Lee BK, RYU HH, Moon JM, Chun BJ, Min YI. Predictors of mortality in hemodynamically unstable traumatic patients with pelvic fracture. J Korean Soc Emerg Med 2009;20:280-7.

17. Burlew CC, Moore EE, Smith WR, Johnson JL, Biffl WL, Barnett CC, et al. Preperitoneal pelvic packing/external fixation with secondary angioembolization: optimal care for life-threatening hemorrhage from unstable pelvic fractures. J Am Coll Surg 2011;212:628-35; discussion 635-7.

18. Costantini TW, Coimbra R, Holcomb JB, Podbielski JM, Catalano R, Blackburn A, et al. Current management of hemorrhage from severe pelvic fractures: results of an American Association for the Surgery of Trauma multi-institutional trial. J Trauma Acute Care Surg 2016;80:717-25; discussion 723-5.

19. Brenner ML, Moore LJ, DuBose JJ, Tyson GH, McNutt MK, Albarado RP, et al. A clinical series of resuscitative endovascular balloon occlusion of the aorta for hemorrhage control and resuscitation. J Trauma Acute Care Surg 2013;75:506-11.

20. Cothren CC, Osborn PM, Moore EE, Morgan SJ, Johnson JL, Smith WR. Preperitoneal pelvic packing for hemodynamically unstable pelvic fractures: a paradigm shift. J Trauma 2007;62:834-42; discussion 839-42.

21. Sokol KK, Black GE, Willey SB, Song MY, Marko ST, Eckert MJ, et al. Preperitoneal balloon tamponade for lethal closed retroperitoneal pelvic hemorrhage in a swine model: a comparable and minimally invasive alternative to open pre-peritoneal pelvic packing. J Trauma Acute Care Surg 2016;81:1046-55. 